\title{
Influences on older people's decision making regarding choice of topical or oral NSAIDs for knee pain: qualitative study
}

\author{
Dawn Carnes, research fellow, ${ }^{1}$ Yasir Anwer, MSc student, ${ }^{1}$ Martin Underwood, professor of general \\ practice, ${ }^{1}$ Geoff Harding, senior research fellow, ${ }^{2}$ Suzanne Parsons, research fellow', on behalf of the TOIB \\ study team
}

${ }^{1}$ Centre for Health Sciences, Barts and The London, Queen Mary University of London, London E1 2AT

${ }^{2}$ Peninsula College of Medicine and Dentistry (Primary Care), Royal Cornwall Hospital, Truro TR1 3LJ

Correspondence to: $\mathrm{M}$ Underwood m.underwood@warwick.ac.uk

doi:10.1136/bmj.39401.699063.BE

\section{ABSTRACT}

Objective To explore the factors that influence older people's decision making regarding use of topical or oral ibuprofen for their knee pain.

Design Qualitative interview study nested within a randomised controlled trial and a patient preference study that compared advice to use oral or topical non-steroidal anti-inflammatory drugs (NSAIDs) for knee pain in older people.

Setting 11 general practices.

Participants 30 people aged $\geq 50$ with knee pain.

Results Participants' decision making was influenced by their perceptions of the associated risk of adverse effects, presence of other illness, nature of their pain, advice received, and practicality. Although participants' understanding of how the medications worked was sometimes poor their decision making about the use of NSAIDs seemed logical and appropriate. Participants model for treatment was to use topical NSAIDs for mild, local, and transient pain and oral NSAIDs for moderate to severe, generalised, and constant pain (in the absence of other more serious illness or risk of adverse effects).

Participants showed marked tolerance and normalisation of adverse effects.

Conclusion Participants had clear ideas about the appropriate use of oral and topical NSAIDs. Taking such views into account when prescribing may improve adherence, judgment of efficacy, and the doctor-patient relationship. Tolerance and normalisation of adverse effects in these patients indicate that closer monitoring of older people who use NSAIDs might be needed.

\section{INTRODUCTION}

Topical and oral non-steroidal anti-inflammatory drugs (NSAIDs) are used to treat knee pain in older people. The different nature and modes of administration mean that patients' preferences and expectations of benefit might need to be part of the decision making process regarding prescribing and use. We carried out a randomised controlled trial and a parallel patient preference study to compare advice to, or the decision to, preferentially use topical or oral NSAIDs, primarily ibuprofen, for knee pain. ${ }^{1-3}$ In both the randomised trial and the preference study outcomes for knee pain were equivalent. In the randomised trial, however, participants in the oral group had more minor adverse effects and possibly more relief from more widespread pain. ${ }^{2}$ In the preference study we found that two to three times more participants chose topical treatment but that those choosing oral treatment seemed to be more tolerant of adverse effects related to NSAID use. ${ }^{2}$ Interpreting these findings in a manner that will inform prescribing decisions is not straightforward. ${ }^{3}$

A model of shared decision making that takes into account both patients' and clinicians' beliefs about clinical benefits, adverse effects, preferences, and costs of NSAIDs might improve care and patients' adherence to prescribed treatment regimens. ${ }^{45}$ Understanding how patients' beliefs determine their preferences for treatment might improve the quality of this shared decision making process and the success of treatment. ${ }^{6}$ For example, factors influencing treatment choices by patients with arthritis include relief of symptoms, the occurrence of adverse effects, and the availability of alternative treatments..$^{7-10}$ The reasons and rationales for these preferences are complex. Patients' understanding and knowledge is built up from various sources, including experience, advice from doctors, and "folk models of illness. ${ }^{1-13}$ Preference for treatment and evaluation of risk might also be influenced by the condition for which the drug is being administered and the perceived level of severity. ${ }^{4}$ These preferences might have affected the choices the participants made about joining the study and which treatment to use and the perceived effectiveness of treatment and toxicity of drugs. ${ }^{14-16}$ To help set the results of that study in context we conducted a nested qualitative study to examine what influenced the decisions of participants about taking part in the study and their use of topical or oral ibuprofen for their knee pain.

\section{METHOD}

Details of methods and the clinical results are available elsewhere. ${ }^{1-3}$ Participants for the qualitative study came 
from 11 practices across the UK where we had relevant research ethics and governance approval. The nested qualitative study comprised two main components.

\section{Rationale behind the decisions}

We explored the rationale behind patients' decisions to take part in the preference study or the randomised trial and, for those in the preference study, their decisions to choose topical or oral medication. We conducted telephone interviews with a purposive sample of participants according to which study they had joined and their choice or allocation of treatment. These interviews lasted about 45 minutes.

Patients' experiences of and beliefs about adverse effects We interviewed a second purposive sample according to the participants' choice of treatment or allocated treatment in the trial and whether it had had an adverse effect. ${ }^{3}$ These interviews took place in the participants' home or at their general practice; they lasted about one hour.

All interviews were conducted after the end of quantitative data collection. We selected two different samples of participants because we expected different themes to arise from interviews about treatment choices and from interviews focused on adverse effects. As initial analysis of the two groups of interviews generated similar themes, we aggregated information from both groups for this analysis.

\section{Participants}

In an initial pilot study we interviewed eight participants from one general practice. The data from these interviews informed the selection criteria for the purposive sample and the topic guide but were not used in the main analysis. In both studies we made a purposive selection according to age, sex, and general

Interview topic guide

Preference for randomised trial $v$ preference study and topical $v$ oral ibuprofen General information about quality of life; and type and history of knee pain Understanding and knowledge about knee pain and medication

Management of knee pain

Motivation to participate in trial

Preferred treatment and why

Attitude to trial and treatment, including compliance

Attitude about the future

Adverse effects from topical or oral ibuprofen

General health and history of knee pain

Management of pain in the past and why

Beliefs about medication and its action

Knowledge about adverse effects of medication

Beliefs about mechanism of action of adverse effects

Reasons for continuing or abstaining from treatment, including compliance

Tolerance of risk practice. All interviews took place after participants had finished the quantitative study.

\section{Interview process}

We developed topic guides from reference to the literature, brainstorming within the research team, and data arising from some exploratory interviews that we conducted in one of our pilot practices (box). DC (an osteopath and health services researcher) undertook the preference interviews and YA (a doctor) undertook the interviews on adverse effects. All the interviews were tape recorded, anonymised, and transcribed.

\section{Data analysis}

We used the "framework" approach to analyse the interview data. ${ }^{17}$ This involved the researchers familiarising themselves with the content of the interview transcripts and then developing a thematic framework by mapping ideas and opinions articulated in the transcripts and conflating these into sub-themes and themes. The framework was applied to the data by coding each section of text to each idea or sub-theme and grouping them into themes. Interview data from each theme and sub-theme was summarised in charts. One chart was developed for each major theme. These charts provided an analytical tool from which emergent concepts could be identified.

DC, YA, and SP undertook the interview coding; $\mathrm{DC}$ carried out the primary analysis of the preference interviews and YA and SP that of the adverse effects interviews. SP and GH triangulated the analysis of the preference interviews, and DC and GH triangulated the analysis of the adverse effects interviews. They did this by examining whether the lead researchers' interpretations of the data were plausible and by offering competing interpretations where appropriate. An additional third party arbitration stage was available (with MU) should there have been any contentious issues, but this was not required.

\section{RESULTS}

We conducted 30 interviews, 15 telephone interviews about medication preference and 15 face-to-face interviews about adverse effects. Eighteen participants were men. Sixteen participants used topical NSAIDs and 14 used oral NSAIDs. We interviewed five participants aged 50-60 years, 16 aged $61-70$, and 10 aged over 70 .

We grouped the 14 sub-themes into five themes: the nature of pain, mechanism of action and resulting effectiveness of medications, risk assessment of adverse effects, practicality of use, and advice and information about NSAIDs (table).

\section{Nature of pain}

Preference was strongly influenced by whether patients' pain was constant or transient. Constant pain was believed to be caused by structural, irreversible damage to bones and cartilage and was therefore considered to require stronger medication such as oral NSAIDs (table). Transient pain was believed to be 


\section{Factors influencing preference and decisions for taking topical or oral medication}

Nature of pain

Perception of effectiveness of medication

Makes it worse, no difference, limited help, major help, cure
"It just seems like l'm throwing stuff at it and nothing is happening" (patient 11) "Well, they seem to work" (patient 15)

Concepts: swelling associated with pain but not cause, inflammation is poorly "Pain is caused by the cartilage disappearing (so the natural padding goes, understood term and rarely used in lay circles, arthritis is seen as diagnosis, causing the pain). I assume it's the end of the bones rubbing together" (patient 9 ) cause and explanation, degeneration and weakness different, genetic inevitability. Causes: loss of cartilage, bits missing, loss of cushioning, bones rubbing together, muscle deterioration weakness, previous injury, overuse, surgery, age, rotting, weight and pressure, cold, heat, gout, pain in other areas

Perceptions about causes of pain

Mechanism of action and resulting perceived effectiveness of topical and oral ibuprofen

"... get a walnut ... there's a nut inside, rotten ... on the outside perfect" (patient 11)
"The bones wear and crunch against one another" (patient 2)

"How does it know to go to your knee?" (patient 4)

"I have no idea how they work" (patients $8,12,14$ )

"It kills pain; it's not a cure" (patient 2)

Little understanding present. Gel is absorbed through skin into knee to deaden pain. Effect local only. Gel is absorbed and lubricates knee. Tablets go into blood via stomach, drug travels around body to knee to deaden pain via nerves. Tablets go into the blood and to brain telling brain to stop recognising pain. No concept of anti-inflammatory action. Refer to drug as Ibuleve or ibuprofen, pain killers, or knee tablets

"It does something to the brain that makes you think you're not having pain" (patient 13)

"The gel lubricates the joint" (patient 3)

"It's absorbed ... it's some sort of painkiller isn't it?" (patient 1)

"I feel as though something's been put back ... like when you take fluid from something and you put fluid back" (patient 13)

"... makes the muscles swell and stops the joints rubbing together" (patient 11) "It's absorbed more easily so it had a faster effect" (patient 4)

Gel: local application makes it faster acting, more specific/effective, less toxic to rest of body; quicker gel is absorbed faster acting it is; gel not as strong as tablets because it doesn't have to go everywhere and be diluted. Tablets: are more toxic than gel, tablets go everywhere regardless, brain directs tablets to knee; all over effect is positive for those with multisite pain, different medication for different pain sites

"I think the cream just does your knee and the tablets go right through you" [better effect] (patient 14)

"You build up a tolerance to the tablets and then you have to go to something stronger" (patient 2)

"It's in the mind, too; the brain takes it to the parts that hurt" (patient 5)

". . . I do it every day twice a day ... because I can feel it wearing off" (patient 3)

"I mean I use them when I get a problem particularly when I'm out walking, and before I go walking" (patient 15)

"If I get really really bad pain I take an ibuprofen tablet like, but I don't take them very often" (patient 8 )

"I sort of erm do it really as soon as it starts up" (patient 7)

"I was just sort of a bit desperate at the time" (patient 7)

"You have it for a long time, it starts to become part of your daily routine" (patient 12)

"Well, it's (tablets) got to help the other bits of me (with pain)" (patient 4)

Knee pain and pain elsewhere

Isolated knee pain; knee pain plus other musculoskeletal pain; knee pain plus other systemic problems

\section{Risk assessment of adverse effects}

"I'd be rubbing the stuff all over me if I had gel" (patient 9)

"I get worries about all the tablets I take" (patient 1)

"If I took ibuprofen every day, l'd have heartburn every day" (patient 8)

"I'm not normally a sicky person ... so I thought I would give things a rest just for a few days" (patient 13)

"Knee pain is nothing compared to my heart problems" (patient 1)

"I think long term in larger doses I think there would be risks" (patient 16)

"You can get addicted to things I think" (patient 6)

Practicality of use

Practicality

Ease and convenience; inconvenience (gel messy, takes time)

"Sometimes it's just not convenient, or you're handling food" (patient 4)

"l'd have to pull my trousers down to put it on, it's not convenient at work" (patient 16)

Advice and information that affected subsequent choice of medication (and consequent trial and trial arm choice)

"If it was a doctor who said, 'We'll try you on so and so', I would try it" (patient 7) knowledge

Concept of preference by default (necessary medically); medical staff superio knowledge; little trust in own knowledge; accept narratives at face value, no matter from whom, no evaluation of knowledge and "blind faith"
By default/lack of

"No, just an ordinary layman off the street, you know like you do when you're talking on the bus" (patient 13)

I'm squeamish about reading about side-effects because I imagine you have them later" (patient 4)

"I've had stomach problems and I couldn't take the ibuprofen" (patient 2) narratives: used to support opinion; previous illness: all relative to severity-for "I've never had any trouble taking tablets [heart, blood pressure]; tablets have example, knee pain to diabetes and heart failure; previous similar medication never been a problem to me" (patient 7) experience

"My friend's husband was so poorly with them" (patient 1)

"I hadn't tried the cream so I thought it would be interesting" (patient 9) 

medication; extra attention and care for their pain
Illustrative quotes

"I was just asked by (practice nurse) if I would just like to, you know, take it" (patient 12)

"I think they knew of me ... I'm a good guinea pig perhaps" (patient 8)

"It was a worthwhile project ... if I could contribute then I ought to do" (patient 15) "I stand a lot, because l'm frightened l'll seize up" (patient 13)

"Ah well, life goes on" (patient 5)

Resignation: nothing of any use-avoidance; acceptable form of controlcontinue use; need more relief-use both; last resort-surgical intervention; exercise-self help; alternative treatment
"I would try resting, then gel, then ibuprofen, then GP, then knee replacement" (patient 10)

"New knees are a last resort" (patient 1) caused by weakness in the knee and personal responsibility for pain-for example, from overuse. Those with transient pain considered their pain less degenerative and thought that topical preparations were preferable.

\section{Mechanism of action and resulting effectiveness of treatment}

Topical preparations were considered to have a localised rather than a generalised effect (table). Participants thought the preparation went through the skin to alleviate pain only in the place where it was applied. The overriding beliefs were that topical preparations would not affect the rest of the body and would take effect more quickly. Participants had clear beliefs about how topical preparations worked, which were enhanced by the visual feedback of the topical preparation disappearing into the skin. They believed that the faster the preparation disappeared, the more effective it was. Topical preparations were assumed to have a lower dose of the active ingredient because it did not have to be "shared" with the rest of the body, and for this reason topical preparations were considered less toxic. This appealed to patients with digestive and other systemic problems and therefore informed their preferences.

Oral preparations were thought to have a general rather than a local effect. Participants were less confident about understanding the mechanism of action of the tablets as they did not have the visual feedback of a topical preparation disappearing into the affected area. The active ingredients of the tablets were thought to travel through the whole body before reaching the knees and therefore oral preparations took longer to take effect than topical ones. Oral preparations were seen as toxic to everywhere but the

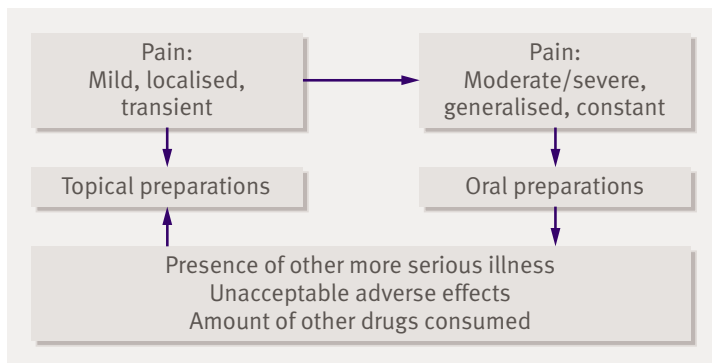

Fig 1 Nature of pain and choice of NSAID preparation knees. There was a contradictory belief that, although the oral drug was considered less powerful because of dilution, by the time it got to the knees it was still more powerful than the topical preparations. Those with multiple sites of pain were happier to take oral preparations because the drug might help other areas while it circulated around the body. Conversely, there was a perception that medication taken for a specific problem had a specific effect-for example, take one tablet for the knee and another for the neck.

Neither route of administration was expected to be a cure. On the whole, topical preparations were considered effective in the short term for mild to moderate knee pain and oral preparations in the medium to long term for severe knee pain (fig 1). The exception was the presence of additional illness. Participants then worried about the number of drugs taken and the interaction between them.

When participants viewed their medication as effective they were happy to carry on with the treatment to manage their knee pain, although the way they self medicated was adapted to their lifestyles. In most cases when the treatment was ineffective participants reported that they would stop taking the drug, although there were a few exceptions. Those that did so said it was because they were in a trial, their general practitioner had told them to take the medication, they hoped that the medication might be effective in the longer term, and there were no other options.

\section{Risk assessment of adverse effects}

If participants believed that their treatment was of benefit to them they were willing to tolerate some adverse effects, such as a rash, fatigue, change in bowel habits, and an occasional upset stomach (table). Nearly all those interviewed were sceptical about whether it was possible to experience adverse effects from topical preparations. Participants believed that only preparations that worked internally could have adverse effects on the rest their body. Topical preparations were viewed as safe because they did not enter the blood system in the same way as tablets and they worked only on the part of the body that was in pain. Oral preparations, however, were considered to be harmful to those parts of the body that were not in pain and in need of treatment. Participants therefore believed that taking oral medication was a higher risk option than using a topical preparation. 


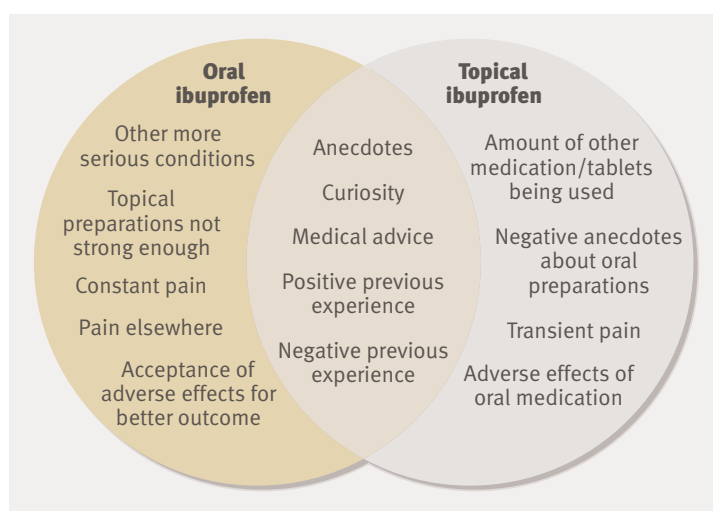

Fig 2 Factors influencing patient preferences for topical or oral ibuprofen

Mild risks were seen as tolerable and acceptable adverse effects. They included things like a rash, an "acidic stomach," or a mild change in bowel habits-for example, diarrhoea once a week. Participants would not tolerate adverse effects if they were continuous and unmanageable; these included things like swelling, headache, dizziness, or visual problems.

Because they were part of a trial and appreciated that every result was important, our participants may have been more willing to tolerate adverse effects even if the effect of the drug was limited. They also tended to "normalise" symptoms by perceiving them as a consequence of "old age," even though these symptoms could be indicators of intolerance to NSAID. Participants did not necessarily communicate these symptoms to their general practitioner. They reported that they would be willing to take additional medication to combat any adverse effects resulting from the NSAIDs, especially if their general practitioner suggested it, they considered it to be a good idea, they were desperate, and the drug helped with their pain.

\section{Practicality of use}

The practicality of taking the drug was a consideration for those in the preference study. Some wanted a "quick fix" and others did not want to deal with the time and "mess" of the topical preparations (table). Those who perceived the medication regimen as contrary to their lifestyle generally viewed their medication less positively and as having less effect on their pain.

\section{Advice and information about NSAIDs}

Advice and "information" about drugs informed preference. This was obtained through consultations and from those with medical knowledge, narratives from others, advertisements, and promotional literature and articles seen in magazines and newspapers (table). The validity and accuracy of information, regardless of source, was rarely questioned. Those with poor understanding of the drug or who did not feel able to make the decision left the responsibility of decision making to their general practitioner, who was assumed to "know best." Patients generally trusted the advice of their general practitioner implicitly; this had the effect of increasing how much they would tolerate adverse effects. We found that participants' comprehension depended on the use of lay terminology and effective face-to-face communication. Few participants remembered the content of written instructions and many actively avoided reading about adverse effects for fear of experiencing them through the power of suggestion. Participants also believed that because they were in a study they would be well looked after and monitored and nothing too untoward would happen because of the increased surveillance.

Those who had a strong preference, but were unsure why, validated their choice as a trade-off between adverse effects, pain relief, and improved function or, in the case of topical medication, a lower risk of adverse effects.

Figure 2 shows the main factors that influenced people's choices about NSAID medication. The diagram shows that factors influencing decision making towards oral preparations were based on perceived severity of their condition and pain elsewhere. Anecdotes, previous experience, and medical advice influenced all participants. Those choosing topical preparations were primarily influenced by their use of other drugs and experience of adverse effects. They tended to have pain that was transient, whereas pain in those choosing oral treatment tended to be constant (see fig 1).

\section{DISCUSSION}

Patients with mild transient knee pain, considered to be caused by weakness or mild degeneration, preferred treatment with topical preparations. Oral medication was preferred by those with more serious, constant, or widespread pain. Our quantitative data also supported this finding. ${ }^{2}$ Participants with more severe pain were more likely to choose an oral preparation and, in the randomised trial, oral preparations seemed to be slightly more effective for widespread pain than topical ones. Exceptions to preferring oral medication were the presence of more serious illness and intolerance of oral treatment. The main issues identified from our emergent concepts were lack of understanding and knowledge and the impact this had on informed choice; trust in the general practitioner's advice and being in a research study; and perception of risk from NSAID use and education about adverse effects.

\section{Participants' lack of understanding and knowledge}

Patients' understanding about pain and the mode of action of treatment was generally limited. Although we thought we had presented adequate information about the study, participants were still unclear about it and about the drugs used. ${ }^{618} 19$ This has implications for preference studies: if patients' understanding is limited, the ability of patients and study participants to make informed decisions about use of medication and participation in a study is questionable. 


\section{Trust and the research process}

Like others who have studied older people, we found that they were relatively trusting and accepting of their general practitioners' advice and decisions about their health care. ${ }^{20}$ In our study, the advice of general practitioners played an important role in the type of medication used. We also found a high level of trust in both the general practitioner and the trial process. In addition, participants tended to normalise general malaise, aches, and lack of wellbeing as a result of "being old" rather than as a consequence of the treatment prescribed. This, coupled with poor understanding of important adverse effects, could lead to an increase in serious adverse effects and unplanned hospital admissions if patients do not recognise minor adverse effects as such. In older people this is compounded by the increased prevalence of comorbidities and the high proportion taking multiple medications. ${ }^{21}$ Complex drug treatment regimens are thought to affect adherence and to contribute to confusion about the drug to which a particular adverse effect is attributable. ${ }^{22}$ Our results add to a growing body of research that indicates a need to monitor elderly patients closely to ensure that reported "normalised" and "accommodated for" adverse effects are really minor. ${ }^{23}$ Older patients who are using NSAIDs should be encouraged to communicate symptoms to their doctor; and closer monitoring for adverse effects may be needed for this. ${ }^{9}$

\section{Perception of risk from NSAIDs}

An expert panel has defined a set of adverse indications strong enough to advise the cessation of NSAID use. ${ }^{3}$ These were mainly clinical markers such as changes in concentrations of haemoglobin, ferritin, or creatinine, lung function, blood pressure, and indigestion. Our study participants reported indigestion, fatigue, and breathlessness but did not necessarily associate them with NSAID use. There seem to be differences between perceptions of practitioners and patients of adverse effects of oral NSAIDs. ${ }^{41024}$ The risk of adverse effects influences choice; patients may often opt for less effective treatments first to avoid the toxicity of other more effective medication, which has implications for how their use and adverse effects are monitored. ${ }^{5}$

\section{Education}

In common with previous researchers we found that increasing patients' knowledge through education about the causes of knee pain, mode of action of treatment, and adverse effects improves both adherence and informed choice. ${ }^{1825}$

\section{Strengths and limitations}

Our sample was selected on the basis of the study they participated in and, for some, the presence of adverse effects. They were all English speaking and predominantly white British. Interviews were conducted both face-to-face and by telephone and covered different topics. We would have preferred to conduct all our

\section{WHAT IS ALREADY KNOWN ON THIS TOPIC}

Non-steroidal anti-inflammatory drugs (NSAIDs) are effective but can produce serious adverse effects

For older patients with knee pain advice to use oral or topical NSAIDs results in equally effective relief

Patients may be prepared to forgo some effectiveness of treatment to avoid adverse effects

\section{WHAT THIS STUDY ADDS}

Choice of topical or oral treatment depends on perceived risk of adverse effects, nature of pain, the presence of other

illness, practicality, and medical advice

General practitioners' and patients' perceptions of the importance of adverse effects from oral NSAIDs may differ

Closer monitoring for potential adverse effects is required

interviews face-to-face but, because this was a nationwide study and researchers did not have enough time to travel, we included participants from seven practices in telephone interviews and from four practices in face-toface interviews. Both approaches produced a large amount and range of quality data, but we have to recognise that this research is based on a fairly healthy pre-screened population of older people with knee pain. However, the trial they participated in was a pragmatic one and the issues raised are congruent with those found in some other studies on this topic. ${ }^{2426}$ But because of the particular nature and characteristics pertinent to older people and patients with chronic pain the results presented may not be generalisable to other drugs or different age groups.

\section{Conclusion}

Patients' decisions about topical or oral treatment for their knee pain were logical and based on the nature of pain, risk of adverse effects, advice, and practicalities. An acceptable lay model for the use of NSAIDs for knee pain is that topical preparations are used for mild, localised, and transient knee pain and oral preparations for more severe, generalised, constant knee pain in the absence of other illness or risk of adverse effects. There is, however, a need to ensure that communication through medical consultations allows practitioners to listen to their patients' needs and to monitor them appropriately for adverse effects. Shared decision making is preferable to encourage adherence to the treatment process and positive perception of the drug used. Because of the equivalence of topical and oral NSAIDs for knee pain, practitioners need to advise the use of preparations that are practical, appropriate, and acceptable to the patient.

We thank Lynette Edwards for comments on earlier drafts of this paper and to all our interviewees for giving up their time for this study. Full details of the study team are given elsewhere. ${ }^{2}$

Contributors: DC conducted the qualitative preference data collection, led the analysis for this study element, contributed to the analysis of the adverse effects data, cowrote the first draft, and contributed to successive drafts. YA conducted the adverse effects interviews, participated in the analysis and interpretation of the qualitative adverse events study, and 
contributed to successive drafts of this paper. GH was one of the origina applicants, designed and conducted the pilot qualitative study, was involved in the analysis of both the preference and adverse effects data, and contributed to successive drafts of this paper. MU was the principal investigator, was primarily responsible for the original grant application, took part in the design of the qualitative study, led the overall study team, and contributed to the interpretation of the data and to successive drafts of this paper. SP was one of the original applicants for the study, designed the qualitative study, contributed to the analysis of the preference data collection, led the analysis of the adverse effects data, and led the production of the first draft of this paper and contributed to successive drafts. SP is guarantor.

Funding: This study was commissioned by the NHS Health Technology Assessment Programme, project reference 01/09/02. Goldshield Pharmaceuticals supplied the starter packs of topical ibuprofen. Competing interests: MU has received speaker fees from Pfizer, the manufacturers of celecoxib.

Ethical approval: Northern and Yorkshire multicentre research ethics committee (MREC 2/3/1)

Provenance and peer review: Not commissioned; externally peer reviewed.

1 Cross PL, Ashby D, Harding G, Hennessy EM, Letley L, Parsons S, et a TOIB Study. Are topical or oral ibuprofen equally effective for the treatment of chronic knee pain presenting in primary care: $a$ randomised controlled trial with patient preference study. BMC Musculoskelet Disord 2005;6:55.

2 Underwood M, Ashby D, Cross P, Hennessy E, Letley L, Martin J, et al. Advice to use topical or oral ibuprofen for chronic knee pain in olde people: randomised controlled trial and patient preference study. BMJ 2007 doi: 10.1136/bmj.39399.656331.25.

3 Underwood MR, Carnes D, Cross P, Harding G, Hennessy E, Letley L, et al. Topical or oral ibuprofen for chronic knee pain in older people (TOIB). Health Technol (in press).

4 Sale JE, Gignac M, Hawker G. How "bad" does the pain have to be? A qualitative study examining adherence to pain medication in older adults with osteoarthritis. Arthritis Rheum 2006 Apr 15;55:272-8.

5 Elliott RA, Ross-Degnan D, Adams AS, Safran DG, Soumerai SB. Strategies for coping in a complex world: adherence behaviour among older adults with chronic illness. J Gen Intern Med 2007;22:805-10.

6 Victor C, Ross F, Axford J. Capturing lay perspective in a randomised control trial of a health promotion intervention for people with osteoarthritis of the knee. J Eval Clin Pract 2004;10:63-70.

7 Clark JP, Hudak PL, Hawker GA, Coyte PC, Mahomed NN, Kreder HJ et al. The moving target: a qualitative study of elderly patients' decision-making regarding total joint replacement surgery. J Bone Joint Surg Am 2004;86-A:1366-74.

8 Toye FM, Barlow J, Wright C, Lamb SE. Personal meanings in the construction of need for total knee replacement surgery. Soc Sci Med 2006;63:43-53.
9 Goodacre L, Goodacre J. Factors influencing the beliefs of patients with rheumatoid arthritis regarding disease modifying medication. Rheumatology (Oxford) 2004;43:583-6.

10 Fraenkel L, Wittink DR, Concato J, Fried T. Informed choice and the widespread use of anti-inflammatory drugs. Arthritis Rheum 2004;51:210-4.

11 Charles C, Gafni A, Whelan T. Decision-making in the physicianpatient encounter: revisiting the shared treatment decision-making model. Soc Sci Med 1999;49:651-61.

12 Thom D, Kravitz R, Bell R, Krupat E, Azari R. Patient trust in the physician: relationship to patient requests. Fam Pract 2002;19:476-83.

13 Borders T, Rohrer J, Xu T, Smith D. Older persons' evaluation of health care: the effects of medical scepticism and worry about health. Health Ser Res 2004;39:35-52.

14 Awad MA, Shapiro SH, Lund LJ, Feine JS. Determinants of patients' treatment preferences in a clinical trial. Community Dent Oral Epidemiol 2000;28:119-25.

15 King M, Nazareth I, Lampe F, Bower P, Chandler M, Morou M, et al. Conceptual framework and systematic review of the effects of participants' and professionals' preferences in randomised controlled trials. Health Technol Assess 2005;9:1-186.

16 Pound P, Britten N, Morgan M, Yardley L, Pope C, Daker-White G, et al. Resisting medicines: a synthesis of qualitative studies of medicine taking. Soc Sci Med 2005;61:133-55.

17 Ritchie J, Spencer L. Qualitative data analysis for applied policy research. In: Bryman A, Burgess RG, eds. Analysing qualitative data London: Routledge, 1994:173-94.

18 Stead M, Eadie D, Gordon D, Angus K. "Hello, hello-it's English speak!": a qualitative exploration of patient understanding of the science of clinical trials. J Med Ethics 2005;31:664-9.

19 Townsend A, Hunt K, Wyke S. Managing multiple morbidity in mid-life: a qualitative study of attitudes to drug use. BMJ 2003;327:837.

20 Herve C, Mullet E, Sorum PC. Age and medication acceptance. Exp Aging Res 2004;30:253-73.

21 Thomas E, Peat G, Harris L, Wilkie R, Croft PR. The prevalence of pain and pain interference in a general population of older adults: crosssectional findings from the North Staffordshire Osteoarthritis Project (NorStOP). Pain 2004;110:361-8.

22 Williams B, Shaw A, Durrant R, Crinson I, Pagliari C, de Lusignan S. Patient perpectives on multiple medication versus combined pills: a qualitative study. Qual J Med 2005;98:885-93.

23 Benson J, Britten N. What effects do patients feel from their antihypertensive tablets and how do they react to them? Qualitative analysis of interviews with patients. Fam Pract 2006;23:80-7.

24 Belcher V, Fried T, Agostini J, Tinetti M. Views of older adults on patient participation in medication related decision making. J Gen Intern Med 2006;21:298-303.

25 Spinewine A, Swine C, Dhillon S, Franklin B, Tulkens P, Wilmotte L, et al. Appropriateness of use of medicines in elderly inpatients: qualitative study. BMJ 2005;331:935.

26 Mitchell HL, Carr AJ, Scott DL. The management of knee pain in primary care: factors associated with consulting the GP and referrals to secondary care. Rheumatology (Oxford) 2006;45:771-6.

Accepted: 7 November 2007 